\title{
Monte Carlo studies of critical behaviour of systems with long-range correlated disoder
}

\author{
V.V.Prudnikov, P.V.Prudnikov, S.V.Dorofeev, V.Yu.Kolesnikov \\ Dept. of Theoretical Physics, \\ Omsk State University, \\ 55a, Pr. Mira, 644077, Omsk, Russia
}

Received October 5, 2004, in final form December 10, 2004

Monte Carlo simulations of the short-time dynamic behaviour are reported for three-dimensional Ising model and XY-model with long-range spatially correlated disorder at criticality, in the case corresponding to linear defects. The static and dynamic critical exponents are computed with the use of the corrections to scaling. The obtained values of the exponents are in a good agreement with results of the field-theoretic description of the critical behaviour of this model in the two-loop approximation and with our results of Monte Carlo simulations of three-dimensional Ising model in equilibrium state.

Key words: Monte Carlo simulations, critical phenomena, critical dynamics, disordered systems, long-range correlated disorder

PACS: $75.50 . L k, 05.50 .+q, 68.35 . R h, 75.40 . C x$

\section{Introduction}

For the recent years, much effort has been devoted to investigation of the critical behaviour of solids containing quenched defects. In most papers considerations have been restricted to the case of point defects with small concentrations so that the defects and corresponding random fields have been assumed to be Gaussian distributed and $\delta$-correlated.

For the first time, in the work of Weinrib and Halperin (WH) [1], we have been offered a model of the critical behaviour of a disordered system in which the correlation function of the random local transition temperature $g(\mathbf{x}-\mathbf{y})=\ll T_{\mathrm{c}}(\mathbf{x}) T_{\mathrm{c}}(\mathbf{y}) \gg$ $-\ll T_{\mathrm{c}}(\mathbf{x}) \gg^{2}$ falls off with distance as a power law $\sim|\mathbf{x}-\mathbf{y}|^{-a}$. They showed that for $a \geqslant d$ long-range correlations are irrelevant and the usual short-range Harris criterion [2] 2-d $\nu_{o}=\alpha_{o}>0$ of the effect of $\delta$-correlated point defects is realized, where $d$ is the spatial dimension, and $\nu_{o}$ and $\alpha_{o}$ are the correlation-length and the specificheat exponents of the pure system. For $a<d$ the extended criterion $2-a \nu_{o}>0$ of the 
effect of disorder on the critical behaviour was established. As a result, a wider class of disordered systems, not only the three-dimensional Ising model with $\delta$-correlated point defects, can be characterized by a new type of critical behaviour. So, for $a<d$ a new long-range (LR) disorder stable fixed point (FP) of the renormalization group recursion relations for systems with a number of components of the order parameter $m \geqslant 2$ was discovered. The critical exponents were calculated in the one-loop approximation using a double expansion in $\varepsilon=4-d \ll 1$ and $\delta=4-a \ll 1$. The correlation-length exponent was evaluated in this linear approximation as $\nu=2 / a$ and it was argued that this scaling relation is exact and also holds in higher order approximation. In the case $m=1$ the accidental degeneracy of the recursion relations in the one-loop approximation did not permit to find LR disorder stable FP, but a change in critical behaviour of the model from short-range (SR) to LR correlation type was predicted for $\delta>\delta_{\mathrm{c}}=2(6 \varepsilon / 53)^{1 / 2}$. Korzhenevskii et al. [3] proved the existence of the LR disorder stable FP for the one-component WH model and also found characteristics of this type of critical behaviour. Also they have considered a very interesting model of the critical behaviour of crystals with LR correlations caused by point defects with degenerate internal degrees of freedom $[3,4]$.

Ballesteros and Parisi [5] have studied by Monte Carlo means the critical behaviour in equilibrium of the 3D site diluted Ising model with LR spatially correlated disorder, in the $a=2$ case corresponding to linear defects. They have computed the critical exponents of these systems with the use of the finite-size scaling techniques and found that a $\nu$ value is compatible with the analitical predictions $\nu=2 / a$.

However, numerous investigations of pure and disordered systems performed with the use of the field-theoretic approach show that the predictions made in the oneloop approximation, especially based on the $\varepsilon$-expansion, can differ strongly from the real critical behaviour [6-9]. Therefore, the results for WH model with LR correlated defects received based on the $\varepsilon, \delta$-expansion $[1,3,4,10-12]$ was questioned in our paper [13], where a renormalization analysis of scaling functions was carried out directly for the 3D systems in the two-loop approximation with the values of $a$ in the range $2 \leqslant a \leqslant 3$, and the FPs corresponding to stability of various types of critical behaviour were identified. The static and dynamic critical exponents in the two-loop approximation were calculated with the use of the Pade-Borel summation technique. The results obtained in [13] essentially differ from the results evaluated by a double $\varepsilon, \delta$-expansion. The comparison of calculated the exponent $\nu$ values and ratio $2 / a$ showed the violation of the relation $\nu=2 / a$, supposed in [1] as exact.

The models with LR-correlated quenched defects have both theoretical interest due to the possibility of predicting new types of critical behaviour in disordered systems and experimental interest due to the possibility of realizing LR-correlated defects in the orientational glasses [14], polymers [15], and disordered solids containing fractal-like defects [3] or dislocations near the sample surface [16].

To shed light on the reason of discrepancy between the results Monte Carlo simulation of the 3D Ising model with LR-correlated disorder [5], in the $a=2$ case and the results our renormalization group description of this model [13], we have computed by the short-time dynamics method $[17,18]$ the static and dynamic critical 
exponents for the same site-diluted 3D Ising model with the linear defects of random orientation in a sample.

We have considered the following Ising model Hamiltonian defined in a cubic lattice of linear size $L$ with periodic boundary conditions:

$$
H=-J \sum_{\langle i, j\rangle} p_{i} p_{j} S_{i} S_{j},
$$

where the sum is extended to the nearest neighbours, $S_{i}= \pm 1$ are the usual $Z_{2}$ spin variables, and the $p_{i}$ are quenched random variables $\left(p_{i}=1\right.$, when the site $i$ is occupied by spin, and $p_{i}=0$, when the site is empty), with LR spatial correlation. An actual $p_{i}$ set will be called a sample from now on. We have studied the next way to introduce the correlation between the $p_{i}$ variables for WH model with $a=2$, corresponding to linear defects. We start with a filled cubic lattice and remove lines of spins until we get the fixed spin concentration $p$ in the sample. We remove lines along the coordinate axes only to preserve the lattice symmetries and equalize the probability of removal for all the lattice points. This model was referred in [5] as the model with non-Gaussian distribution noise. In contrast to [5] we put a condition of linear defects disjointness on their distribution in a sample, whereas in [5] the possibility of linear defects intersection is not discarded. The condition of linear defects disjointness corresponds to WH model since the intersection of linear defects being taken into consideration results in additional vertixes of interaction which are absent in the effective Hamiltonian of WH model.

In this paper we have investigated the systems with the spin concentration $p=$ 0.8. We have considered the cubic lattices with linear sizes $L$ from 16 to 128 . The Metropolis algorithm has been used in simulations.

A lot of results have been recently obtained concerning the critical dynamical behaviour of statistical models $[17,18]$. This kind of investigation was motivated by analitical and numerical results contained in the papers of Janssen et al [19] and Huse [20]. In this paper we consider only the dynamics of model A in the classification of Hohenberg and Halperin [21]. The Metropolis Monte Carlo scheme of simulation with the dynamics of a single-spin flips reflects the dynamics of model A and enables us to compare the obtained dynamic critical exponent $z$ with the results of our renormalization group description of critical dynamics of this model [13] having LR-disorder. In general, to determine the dynamic exponent $z$ and the static exponents, a dynamic process that starts from a completely ordered state with the spins oriented in the same direction is more favorable, since fluctuations are much smaller. For a large enough lattice in the critical range, the dynamic scaling form of the magnetization is written as

$$
M(t, \tau)=t^{-\beta / \nu z} F\left(t^{1 / \nu z} \tau\right)
$$

where $t$ is the dynamic evolution time, $\tau=\left(T-T_{\mathrm{c}}\right) / T_{\mathrm{c}}$ is the reduced temperature, and $\beta, \nu$ are the well-known static critical exponents. If $\tau=0$, the magnetization decays by a power law

$$
M(t) \sim t^{-\beta / \nu z}
$$


If $\tau \neq 0$, the power law behaviour is modified by the scaling function $F\left(t^{1 / \nu z} \tau\right)$. From this fact, the critical temperature $T_{\mathrm{c}}$ and the critical exponent $\beta / \nu z$ can be determined. In figure 1 the magnetization $M(t)$ for samples with linear size $L=$ 128 at $T=3.919,3.925,3.930,3.935$ and 3.940 is plotted in double-log scale. The resulting curves in figure 1 have been obtained by averaging over 3000 samples with different linear defect configurations. We have determined the critical temperature $T_{\mathrm{c}}=3.930(2)$ from the best fitting of these curves by power law.

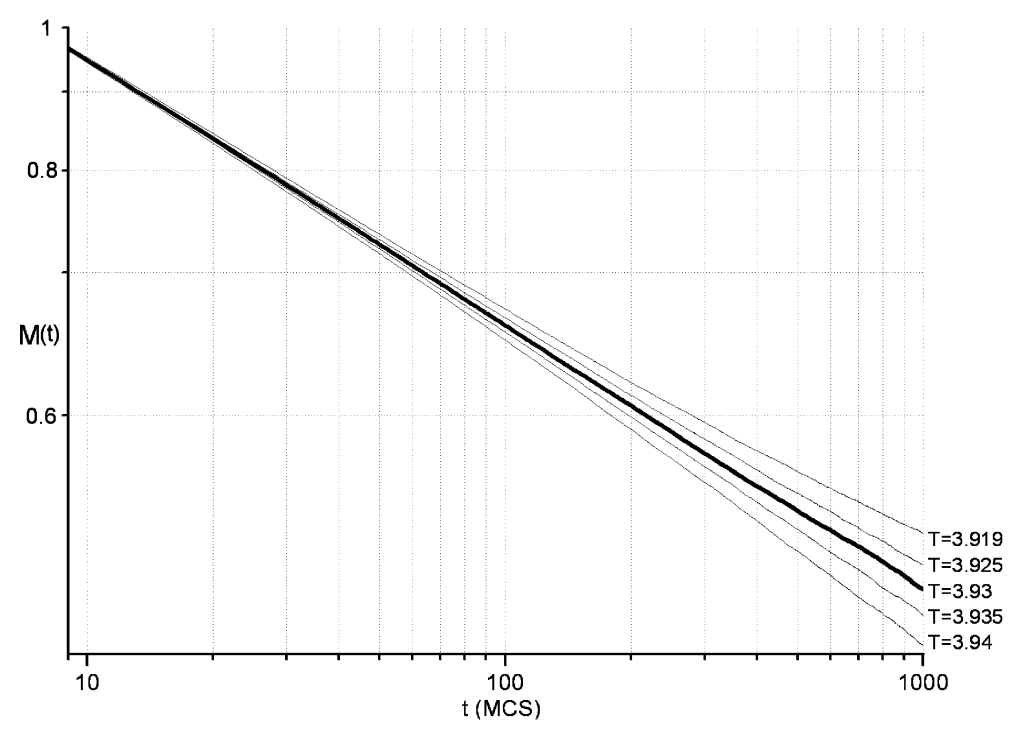

Figure 1. Time evolution of the magnetization $M(t)$ for $L=128$ and for different values of the temperature $T$.

The critical temperature determined in [5] for the same system with spin concentration $p=0.8$ in the non-Gaussian case is $T_{\mathrm{c}}=3.8891(2)$. This difference of the critical temperature values shows that different principles of distribution of linear defects are the reason of discrepancy between the results obtained in [5] by Monte Carlo simulation of the 3D Ising model with LR-correlated disorder, and results in renormalization group description of this model [13].

In order to check-up the critical temperature value independently, we have carried out in equilibrium the calculation of Binder cumulant $U_{4}$, defined as

$$
U_{4}=\frac{1}{2}\left(3-\frac{\left[\left\langle M^{4}\right\rangle\right]}{\left[\left\langle M^{2}\right\rangle\right]^{2}}\right),
$$

and the correlation length

$$
\begin{aligned}
\xi & =\frac{1}{2 \sin (\pi / L)} \sqrt{\frac{\chi}{F}-1} \\
\chi & =\frac{1}{N_{\text {spin }}}\left[\left\langle M^{2}\right\rangle\right] \\
F & =\frac{1}{N_{\text {spin }}}[\langle\Phi\rangle],
\end{aligned}
$$




$$
\Phi=\frac{1}{3} \sum_{j=1}^{3}\left(\left|\sum_{x} S_{x} \exp \left(2 \pi i x_{j}\right)\right|^{2}\right)
$$

where the angle brackets stand for statistical averaging and the square brackets are for averaging over the different impurity configurations. The cumulant $U_{4}(L, T)$ has a scaling form

$$
U_{4}(L, T)=u\left(L^{1 / \nu}\left(T-T_{\mathrm{c}}\right)\right) .
$$

The scaling dependence of the cumulant makes it possible to determine the critical temperature $T_{\mathrm{c}}$ from the coordinate of the points of intersections of the curves specifying the temperature dependence $U_{4}(L, T)$ for different $L$. In figure $2 \mathrm{a}$ the computed curves of $U_{4}(L, T)$ are presented for lattices with sizes $L$ from 16 to 128 . As a result it was determined that the critical temperature is $T_{\mathrm{c}}=3.9275(5)$. In this case for simulations we have used the Wolff single-cluster algorithm with elementary MCS step as 5 cluster flips. We discard 10000 MCS for equilibration and then measure after every MCS with the averaging over 100000 MCS. The results have been averaged over 15000 different samples for lattices with sizes $L=16,32$ and over 10000 samples for lattices with sizes $L=64,128$.

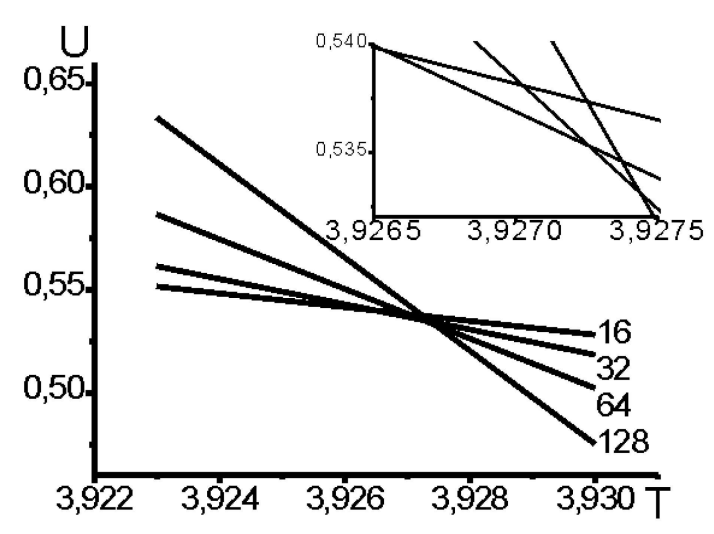

(a)

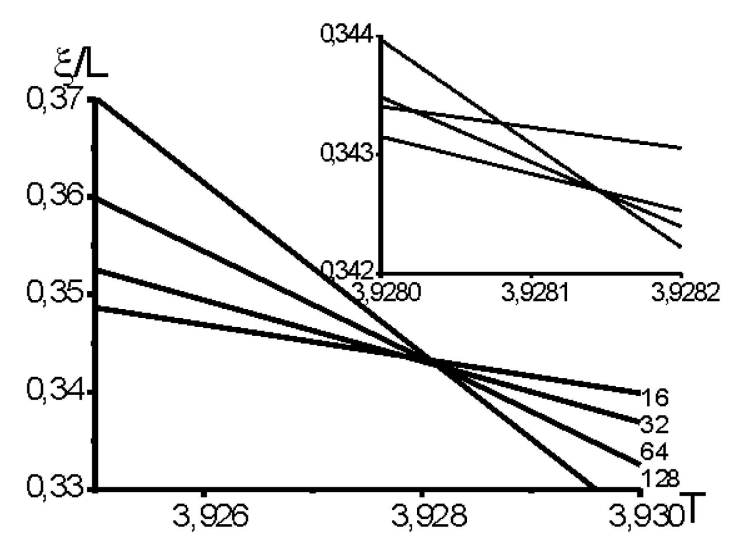

(b)

Figure 2. Binder cumulant $U_{4}(T, L)$ (a) and ratio $\xi / L$ (b) as a function of $\mathrm{T}$ for lattices with different sizes $L$.

In figure $2 \mathrm{~b}$ the computed curves of temperature dependence of ratio $\xi / L$ are presented for lattices with the same sizes, the coordinate of the points of intersections of which also gives the critical temperature $T_{\mathrm{c}}=3.9281(1)$.

Also, we have determined the temperature of intersection of the curves specifying the temperature dependence cumulants $U_{4}(L, T)$ for $L=16$ and $L=32$ with the use of linear defects distribution in samples as in [5] with the possibility of their intersection. Computation gives $T_{\mathrm{c}}(L)=3.8884(6)$ in this case which corresponds to the results in [5] but differs from $T_{\mathrm{c}}(L)=3.9185(5)$ obtained with the use of condition of linear defects disjointness for lattices with the same sizes. 


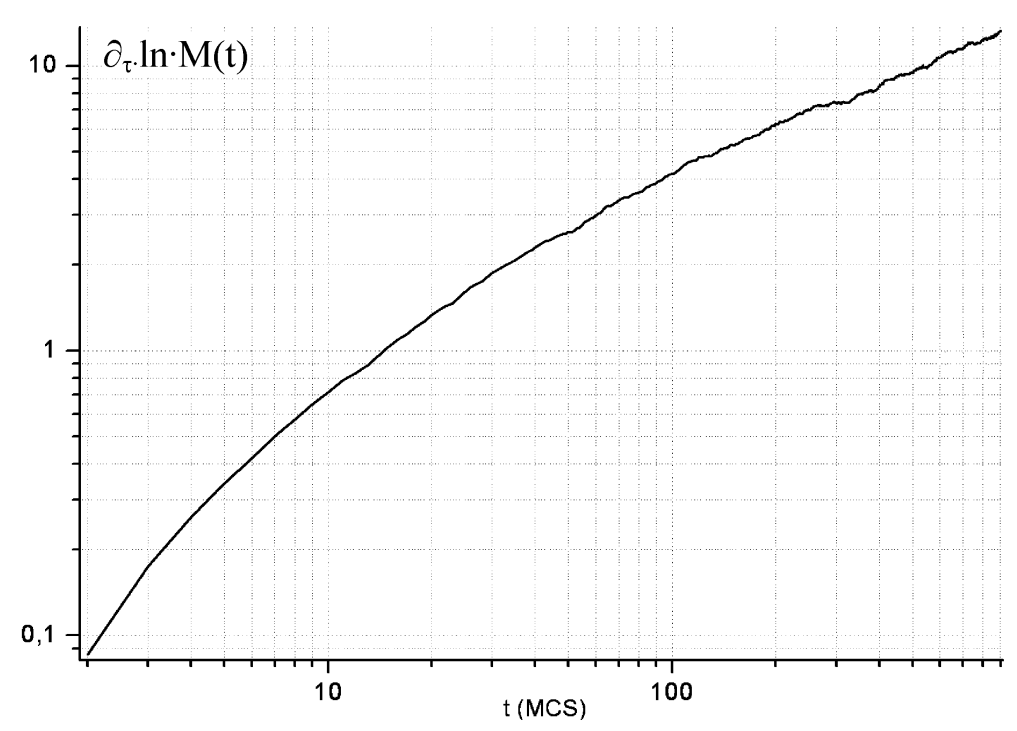

Figure 3. Time evolution of logarithmic derivative of the magnetization $\left.\partial_{\tau} \ln M(t, \tau)\right|_{\tau=0}$ for $L=128$ at the critical temperature $T_{\mathrm{c}}=3.9281$.

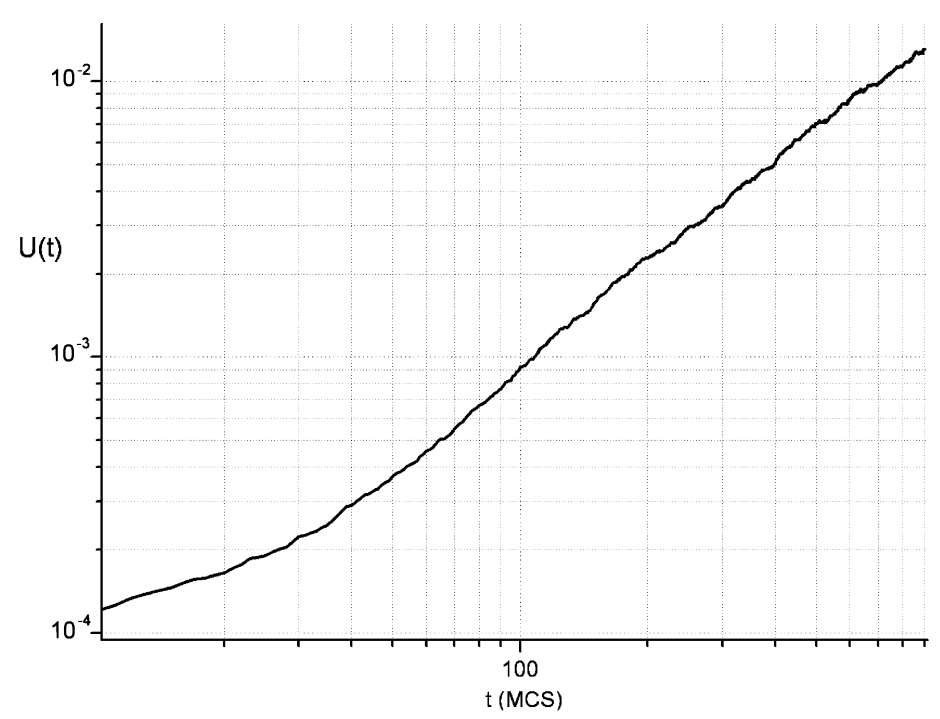

Figure 4. Time evolution of the cumulant $U_{2}(t)$ for $L=128$ at the critical temperature $T_{\mathrm{c}}=3.9281$.

Turning back to short-time dynamics method, we note that the exponent $1 / \nu z$ can be determined if we differentiate $\ln M(t, \tau)$ with respect to $\tau$

$$
\left.\partial_{\tau} \ln M(t, \tau)\right|_{\tau=0} \sim t^{1 / \nu z} .
$$

In order to estimate the dynamic exponent $z$ independently, a second order cumulant $U_{2}=\left[\left\langle M^{2}\right\rangle\right] /[\langle M\rangle]^{2}-1$ can be introduced, and finite size scaling analysis shows

$$
U_{2}(t, L) \sim t^{d / z}
$$


where $d$ is a system dimension. In figure 3 and in figure 4 the logarithmic derivative of the magnetization $\left.\partial_{\tau} \ln M(t, \tau)\right|_{\tau=0}$ with respect to $\tau$ and the cumulant $U_{2}(t)$ for samples with linear size $L=128$ at $T_{\mathrm{c}}=3.9281$ are plotted in log-log scale. The $\left.\partial_{\tau} \ln M(t, \tau)\right|_{\tau=0}$ have been obtained from a quadratic interpolation between the three curves of time evolution of the magnetization for the temperatures $T=3.9250,3.9281,3.9310$ and taken at the critical temperature $T_{\mathrm{c}}=3.9281$. The resulting curves have been obtained by averaging over 3000 samples.
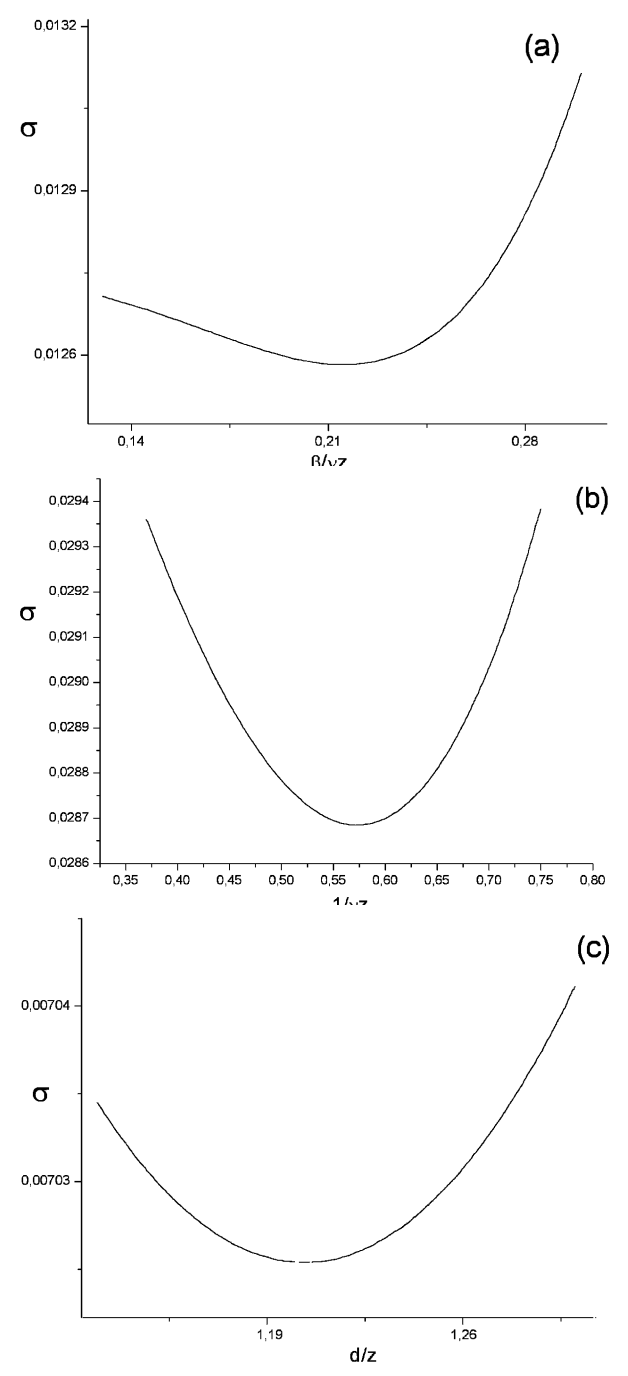

Figure 5. Dependence of the mean square errors $\sigma$ of the fits for the magnetization (a), logarifmic derivative of the magnetization (b), and the cumulant (c) as a function of the exponents $\beta / \nu z, 1 / \nu z$, and $d / z$ for $\omega=0.8$.

We have analysed the time dependence of the cumulant $U_{2}(t)$ and clarified that in the time interval $[50,150]$ the $U_{2}(t)$ is best fitted by power law with the dynamic exponent $z \simeq 2.02$, corresponding to the pure Ising model [22], and the linear defects are developed for $t>400$ MCS only. We have taken into account these dynamic crossover effects for the analysis of the time dependence of magnetization and its 
derivative. Also, we have considered the corrections to the scaling in order to obtain accurate values of the critical exponents. We have applied the following expression for the observables $X(t)$ :

$$
X(t) \sim t^{\Delta}\left(1+A_{x} t^{-\omega / z}\right)
$$

where $\omega$ is a well-known exponent of corrections to scaling. This expression reflects the scaling transformation in the critical range of time-dependent corrections to scaling in the form of $t^{-\omega / z}$ to the usual form of corrections to scaling $\tau^{\omega \nu}$ in equilibrium state for time $\mathrm{t}$ comparable with the order parameter relaxation time $t_{\mathrm{r}} \sim \xi^{z} \Omega(k \xi)$ [21]. Field-theoretic estimate of the $\omega$ value gives $\omega \simeq 0.80$ in the two-loop approximation [15]. Monte Carlo study of Ballesteros and Parisi [5] shows that $\omega \simeq 1.0$.

Table 1. Values of the exponents $\beta / \nu z, 1 / \nu z, d / z$, and minimal values of the mean square errors $\sigma$ in fits for different values of the exponent $\omega$.

\begin{tabular}{|c|c|c|c|c|c|c|}
\hline$\omega$ & $\beta / \nu z$ & $\sigma$ & $1 / \nu z$ & $\sigma$ & $d / z$ & $\sigma$ \\
\hline 0.7 & 0.2112 & 0.0100 & 0.556 & 0.0053 & 1.183 & 0.0100 \\
0.8 & 0.2096 & 0.0088 & 0.559 & 0.0049 & 1.205 & 0.0100 \\
0.9 & 0.2101 & 0.0093 & 0.553 & 0.0070 & 1.213 & 0.0099 \\
1.0 & 0.2090 & 0.0095 & 0.558 & 0.0072 & 1.227 & 0.0098 \\
\hline
\end{tabular}

To analyse our sumulation date we have used the linear approximation of the $\left(X t^{-\Delta}\right)$ on $t^{-\omega / z}$ with the changing values of the exponent $\Delta$ and the exponent $\omega$ from the interval $[0.7,1.0]$. Then, we have investigated the dependence of the mean square errors $\sigma$ of this fitting procedure for the function $X t^{-\Delta}\left(t^{-\omega / z}\right)$ on the changing $\Delta$ and $\omega$. In figure 5 we plot the $\sigma$ for the magnetization (figure $5 \mathrm{a}$ ), logarifmic derivative of the magnetization (figure $5 \mathrm{~b}$ ), and the cumulant (figure $5 \mathrm{c}$ ) as a function of the exponents $\beta / \nu z, 1 / \nu z$, and $d / z$ for $\omega=0.8$. Minimum of $\sigma$ determines the exponents $z, \nu$, and $\beta$ for every $\omega$. In table 1 we present the computed values of the exponents $\beta / \nu z, 1 / \nu z$, and $d / z$, and minimal values of the mean square errors $\sigma$ in these fits for the values of the exponent $\omega=0.7,0.8,0.9,1.0$. We see that the values of $\beta / \nu z, 1 / \nu z$, and $d / z$ are weakly dependent on the change of the exponent $\omega$ in the interval $[0.7,1.0]$, but the $\omega=0.8$ is preferable because it gives the best fit for the magnetization and the logarifmic derivative of the magnetization dates. Finally, for the $\omega=0.8$ we find the following values of the exponents

$$
\begin{aligned}
& z=2.489 \pm 0.021, \\
& \nu=0.719 \pm 0.022, \\
& \beta=0.375 \pm 0.045 .
\end{aligned}
$$

It is interesting to compare these values of exponents with those obtained in [13] with the use of the field-theoretic approach

$$
\begin{aligned}
& z=2.495, \\
& \nu=0.716, \\
& \beta=0.350,
\end{aligned}
$$


which demonstrate a very good agreement with each other, but show an essential difference from Monte Carlo results of Ballesteros and Parisi [5] with $\nu=1.009(13)$ and $\beta=0.526(15)$.

Also, we have carried out the Monte Carlo study of the effect of LR-correlated quenched defects on the critical behaviour of 3D XY-model characterized by the two-component order parameter. As is well-known, renormalization group analysis predicts the possibility of new type of critical behaviour for this model different from critical behaviour of pure XY-like systems or systems with $\delta$-correlated defects. We considered the same site-diluted cubic lattices with the linear defects of random orientation in the samples with the spin concentration $p=0,8$. The critical temperature $T_{\mathrm{c}}=1.875(1)$ was determined by the calculation of Binder cumulant $U_{4}(L, T)$ for lattices with sizes $L$ from 16 to 64 . For simulations we have used the Wolff single-cluster algorithm. Then, we have computed for this critical temperature the static and dynamic critical exponents by the short-time dynamics method through finite size scaling analysis of time evolution of the magnetization $M(t, L)$, the logarithmic derivative of the magnetization $\left.\partial_{\tau} \ln M(t, \tau)\right|_{\tau=0}$, and the second order cumulant $U_{2}(t, L)$. As a result of this analysis we obtained the following values of critical exponents

$$
\begin{aligned}
& z=2.364 \pm 0.007, \\
& \nu=0.778 \pm 0.026, \\
& \beta=0.400 \pm 0.030 .
\end{aligned}
$$

The comparison of these values of exponents with those obtained in [13] with the use of the field-theoretic approach $z=2.365, \nu=0.760$, and $\beta=0.366$ shows their good agreement within the limits of statistical errors of simulation and numerical approximations.

The obtained results confirm the strong effect of LR-correlated quenched defects on both the critical behaviour of 3D Ising model and the systems characterized by the many-component order parameter.

The short-time dynamics method applied in this work has been so far approved of regarding the investigation of the critical behaviour of pure systems only. With the aim to verify the method and the results obtained we also carried out the study of the critical behaviour of 3D Ising model with the linear defects of random orientation by traditional Monte Carlo simulation methods in equilibrium state. For simulations we have used the Wolf single-cluster algorithm. We have computed for the critical temperature $T_{\mathrm{c}}=3.9281(1)$ the values of different thermodynamic and correlation functions in equilibrium state such as the magnetization, susceptibility, correlation length, heat capacity, and Binder cumulant $U_{4}$ for lattices with sizes $L$ from 16 to 128 and the same spin concentration $p=0,80$. The use of well-known scaling critical dependences for these thermodynamic and correlation functions

$$
\begin{aligned}
C(L) & \sim L^{\alpha / \nu}\left(1+a L^{-\omega}\right) \\
M(L) & \sim L^{-\beta / \nu}\left(1+b L^{-\omega}\right), \\
\chi(L) & \sim L^{\gamma / \nu}\left(1+c L^{-\omega}\right)
\end{aligned}
$$




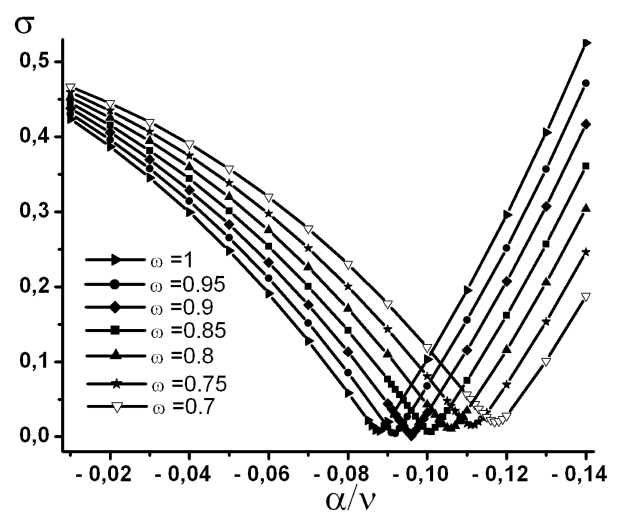

(a)

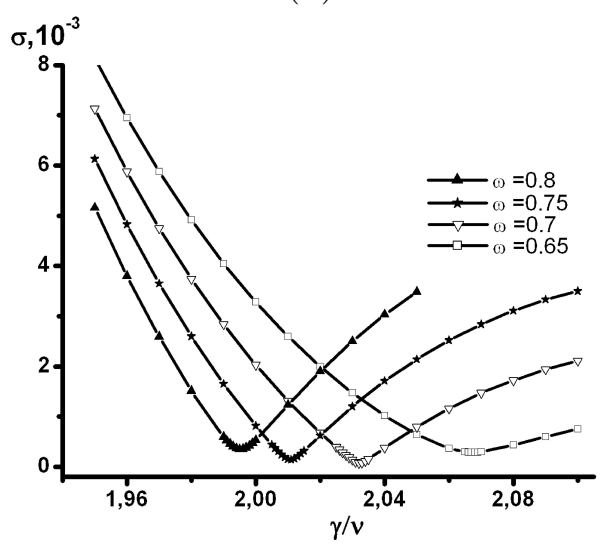

(c)

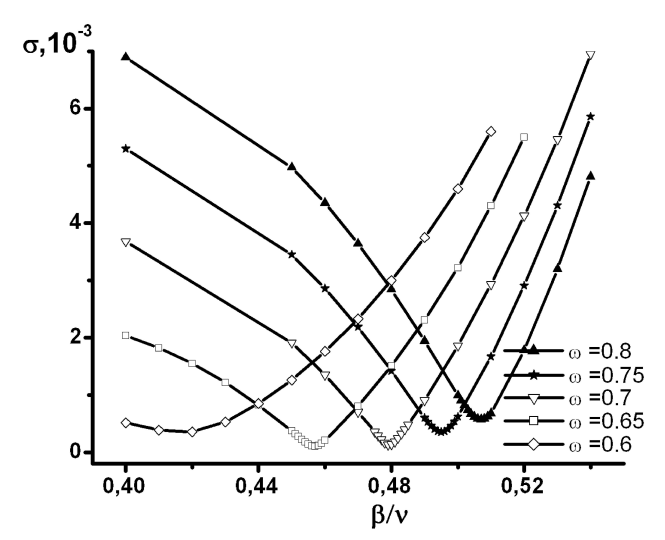

(b)

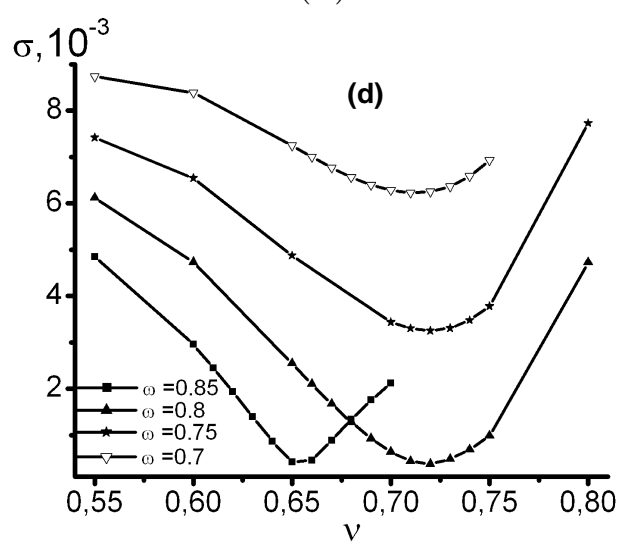

(d)

Figure 6. Dependence of the mean square errors $\sigma$ of the fits for heat capacity (a), magnetization (b), susceptibility (c), and thermal derivative of cumulant (d) as a function of the exponents $\alpha / \nu, \beta / \nu, \gamma / \nu$, and $\nu$ for different values of $\omega$.

$$
\frac{\mathrm{d} U}{\mathrm{~d} T}(L) \sim L^{1 / \nu}\left(1+d L^{-\omega}\right)
$$

makes it possible to determine the critical exponents $\alpha, \nu, \beta, \gamma$, and $\omega$ by means of statistical data processing of simulation results. To analyse sumulation data we have used the linear approximation of the $\left(X L^{-\Delta}\right)$ on $L^{-\omega}$ and then investigated the dependence of the mean square errors $\sigma$ of this fitting procedure for the function $X L^{-\Delta}\left(L^{-\omega}\right)$ on the changing exponent $\Delta$ and $\omega$ values. In figure 6 we plot the $\sigma$ for heat capacity (figure 6a), magnetization (figure 6b), susceptibility (figure 6c), and temperature derivative of cumulant (figure $6 \mathrm{~d}$ ) as a function of the exponents $\alpha / \nu$, $\beta / \nu, \gamma / \nu$, and $\nu$ for different values of $\omega$. Minimum of $\sigma$ determines the values of exponents. In table 2 we present the obtained values of the exponents $\alpha / \nu, \beta / \nu, \gamma / \nu$, $\nu$, and $\omega$, which give minimal values of $\sigma$ in these fits. Then we determine the average value of $\omega=0.76(5)$ with the use of which there were computed the final values of exponents. In table 3 there are presented the values of the exponents obtained in this work by simulation methods and from [13] with the use of the field-theoretic 
approach and scaling relations for critical exponents. The comparison of these values shows their good agreement within the limits of statistical errors of simulation and numerical approximations and good agreement with the values of the static critical exponents computed by the short-time dynamics method. Consequently, the results of the Monte Carlo investigations allow us to recognize that the short-time dynamics method is reliable for the study of the critical behaviour of the systems with quenched disorder and is the alternative to traditional Monte Carlo methods. We are planning to continue the Monte Carlo study of critical behaviour of the model with LR-disorder for different values of spin concentration $p$ and investigate the universality of critical behaviour of diluted systems with LR-disorder focusing on the problem of disorder independence of asymptotic characteristics.

Table 2. Values of the exponents $\alpha / \nu, \beta / \nu, \gamma / \nu$, and $\nu$ with values of the exponent $\omega$, giving the best fit in approximation procedure

\begin{tabular}{|c|c|c|c|c|}
\hline & $\alpha / \nu$ & $\beta / \nu$ & $\gamma / \nu$ & $\nu$ \\
\hline & $-0.096(3)$ & $0.457(2)$ & $2.032(1)$ & $0.710(10)$ \\
\hline$\omega$ & 0.90 & 0.65 & 0.70 & 0.80 \\
\hline
\end{tabular}

Table 3. Values of the critical exponents obtained in present work for average value of exponent $\omega=0,76$ and corresponding exponents from [13]

\begin{tabular}{|c|c|c|c|c|}
\hline & $\alpha$ & $\beta$ & $\gamma$ & $\nu$ \\
\hline present & $-0.078(30)$ & $0.362(20)$ & $1.441(15)$ & $0.710(10)$ \\
\hline$[13]$ & -0.1048 & 0.3504 & 1.4453 & 0.7155 \\
\hline
\end{tabular}

\section{Acknowledgements}

This work was supported in part by the Russian Foundation for Basic Research through Grants No. 04-02-17524 and No. 04-02-39000, and the Ministry of Education and Science of Russian Federation through Grant No. E02-3.2-196.

We are grateful to Yurii Holovatch for the opportunity to contribute to the Festschrift dedicated to the 60th birthday of Reinhard Folk.

We congratulate Prof. Reinhard Folk with jubilee and wish him a good health and happiness.

\section{References}

1. Weinrib A., Halperin B.I., Phys. Rev. B, 1983, 27, 413.

2. Harris A.B., J.Phys. C: Solid State Phys., 1974, 7, 1671.

3. Korzhenevskii A.L., Luzhkov A.A., Schirmacher W., Phys. Rev. B, 1994, 50, 3661. 
4. Korzhenevskii A.L., Luzhkov A.A., Heuer H.-O., Europhys. Lett., 1995, 32, 19.

5. Ballesteros H.G., Parisi G., Phys. Rev. B, 1999, 60, 912.

6. Jug G., Phys. Rev. B, 1983, 27, 609.

7. Mayer I.O., J.Phys. A: Math. Gen., 1989, 22, 2815.

8. Prudnikov V.V., Belim S.V., Ivanov A.V., Osintsev E.V., Fedorenko A.A., Sov. Phys. - JETP, 1998, 87, 527.

9. Prudnikov V.V., Prudnikov P.V., Fedorenko A.A., Sov. Phys. - JETP Lett., 1998, 68, 950.

10. Dorogovtsev S.N., J.Phys. A: Math. Gen., 1984, 17, L677.

11. Korucheva E., Uzunov D., Phys. Status Solidi (b), 1984, 126, K19.

12. Korucheva E., de la Rubia F.J., Phys. Rev. B, 1998, 58, 5153.

13. Prudnikov V.V., Prudnikov P.V., Fedorenko A.A., Phys. Rev. B, 2000, 62, 8777.

14. Binder K., Regir J.D., Adv. Phys., 1992, 41, 547.

15. Blavats'ka V., von Ferber C., Holovatch Yu., Phys. Rev. B, 2001, 64, 041102.

16. Altarelli M., Nunez-Regueiro M.D., Papoular M., Phys. Rev. Lett., 1995, 74, 3840.

17. Zheng B., Int. J. Mod. Phys. B, 1998, 12, 1419.

18. Jaster A., Mainville J., Schulke L., Zheng B., J. Phys. A, 1999, 32, 1395.

19. Janssen H.K., Schaub B., Schmittmann B., Z. Phys. B, 1989, 73, 539.

20. Huse D., Phys. Rev. B, 1989, 40, 304.

21. Hohenberg P.C., Halperin B.I., Rev. Mod. Phys., 1977, 49, 435.

22. Prudnikov V.V., Ivanov A.V., Fedorenko A.A., Sov. Phys. - JETP Lett., 1997, 66, 835.

\title{
Монте Карло дослідження систем з далекосяжно скорельованим безладом
}

\author{
В.В.Прудніков, П.В.Прудніков, С.В.Дорофєєв, \\ В.Ю.Колєсніков
}

Кафедра теоретичної фізики,

Омський державний університет,

55a, Пр. Миру, 644077, Омськ, Росія

Отримано 5 жовтня 2004 р., в остаточному вигляді 10 грудня 2004 p.

Приводяться Монте Карло симуляції коротко-часової динамічної поведінки для тривимірної моделі Ізинга та XY-моделі 3 просторовим далекосяжно скорельованим безладом в критичній області, що відповідає лінійним дефектам. Обчислено статичні та динамічні показники з поправками до скейлінгу. Отримані значення показників добре узгоджуються 3 результатами теоретико-польового опису критичної поведінки цієї моделі в двопетлевому наближені та 3 нашими результатами Монте Карло симуляцій тривимірної моделі Ізинга в рівноважному стані.

Ключові слова: Монте Карло симуляції, критичні явища, критична динаміка, невпорядковані системи, далекосяжний скорельований безлад

PACS: $75.50 . L k, 05.50 .+q, 68.35 . R h, 75.40 . C x$ 